\title{
QUEEN'S
UNIVERSITY
BELFAST
}

\section{Ion Association in [bmim][PF6]/Naphthalene Mixtures: An Experimental and Computational Study}

Del Popolo, M., Mullan, C., Holbrey, J., Hardacre, C., \& Ballone, P. (2008). Ion Association in

[bmim][PF6]/Naphthalene Mixtures: An Experimental and Computational Study. Journal of the American Chemical Society, 130(22), 7032-7041. https://doi.org/10.1021/ja710841n

Published in:

Journal of the American Chemical Society

Queen's University Belfast - Research Portal:

Link to publication record in Queen's University Belfast Research Portal

\section{General rights}

Copyright for the publications made accessible via the Queen's University Belfast Research Portal is retained by the author(s) and / or other copyright owners and it is a condition of accessing these publications that users recognise and abide by the legal requirements associated with these rights.

Take down policy

The Research Portal is Queen's institutional repository that provides access to Queen's research output. Every effort has been made to ensure that content in the Research Portal does not infringe any person's rights, or applicable UK laws. If you discover content in the Research Portal that you believe breaches copyright or violates any law, please contact openaccess@qub.ac.uk. 


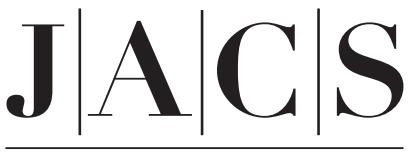

A R T I C L E S

Published on Web 05/08/2008

\title{
Ion Association in [bmim] $\left[\mathrm{PF}_{6}\right] /$ Naphthalene Mixtures: An Experimental and Computational Study
}

\author{
M. G. Del Pópolo, ${ }^{\dagger}$ C. L. Mullan,${ }^{\ddagger}$ J. D. Holbrey ${ }^{\ddagger}$ C. Hardacre,${ }^{\ddagger}$ and P. Ballone ${ }^{\star, \dagger}$ \\ Atomistic Simulation Centre, Queen's University Belfast, Belfast BT7 1NN, U.K., School of \\ Chemistry and Chemical Engineering, Queen's University Belfast, Belfast BT9 5AG, U.K.
}

Received December 5, 2007; E-mail: p.ballone@qub.ac.uk

\begin{abstract}
Mixtures of room temperature ionic liquids (IL) with neutral organic molecules provide a valuable testing ground to investigate the interplay of the ionic and molecular-dipolar state in dense Coulomb systems at near ambient conditions. In the present study, the viscosity $\eta$ and the ionic conductivity $\sigma$ of $1-n$-butyl3-methylimidazolium hexafluorophosphate $\left(\left[b_{m i m}\right]\left[\mathrm{PF}_{6}\right]\right) /$ naphthalene mixtures at $T=80{ }^{\circ} \mathrm{C}$ have been measured at 10 stoichiometries spanning the composition range from pure naphthalene to pure [bmim] $\left.\mathrm{PF}_{6}\right]$. The viscosity grows nearly monotonically with increasing IL mole fraction $(x)$, whereas the conductivity per ion displays a clear peak at $x \approx 15 \%$. The origin of this maximum has been investigated using molecular dynamics simulations based on a classical force field. Snapshots of the simulated samples show that the conductivity maximum is due to the gradual transition in the IL component from an ionic state at high $x$ to a dipolar fluid made of neutral ion pairs at low $x$. At concentrations $x<0.20$ the ion pairs condense into molecular-thin filaments bound by dipolar forces and extending in between nanometric droplets of IL. These results are confirmed and complemented by the computation of dynamic and transport properties in $[\mathrm{bmim}]\left[\mathrm{PF}_{6}\right] /$ naphthalene mixtures at low IL concentration.
\end{abstract}

\section{Introduction}

The vast number of molecular electrolytes collectively known as room temperature ionic liquids (or ionic liquids (ILs) for short) are currently the subject of a massive and concerted research effort because of their recognized potential as environmentally friendly solvents for chemical synthesis, separation technology, and electrochemistry. ${ }^{1}$ Not surprisingly, most of this effort has been devoted to specific applications and to chemicalphysics properties directly related to industrial processes. ${ }^{2}$ It is increasingly apparent, however, that ILs exhibit a range of behaviors that make them remarkable also for more fundamental reasons.

As their name implies, ILs share with traditional salts such as alkali-halides the property of being made of ionic building blocks that in the liquid state or in solution give rise to a steady ionic current whenever a uniform electrostatic field is applied across the system. In turn, this implies that ILs as well as traditional salts display a number of structural, thermodynamic, and dynamical properties arising from the high strength of the Coulomb interaction, and, even more, from its long-range., Prominent among these properties are the regular charge

$\dagger$ Atomistic Simulation Centre.

* School of Chemistry and Chemical Engineering.

(1) (a) Seddon, K. R. Nat. Mater. 2003, 2, 363-365. (b) Welton, T. Chem. Rev. 1999, 99, 2071-2084. (c) Zhao, D. B.; Wu, M.; Kou, Y.; Min, E. Catal. Today 2002, 74, 157. (d) Davis, J. H. Chem. Lett. 2004, 33, 1072. (e) Welton, T. Coord. Chem. Rev. 2004, 248, 2459. (f) Dupont, J.; Suarez, P. A. Z. Phys. Chem. Chem. Phys. 2006, 8, 2441. (g) Endres, F.; El Abedin, S. Z. Phys. Chem. Chem. Phys. 2006, 8, 2101. (h) Parvulescu, V. I.; Hardacre, C. Chem. Rev 2007, 107, 2615.

(2) Rogers, R. D., Seddon, K. R., Eds. Ionic Liquids: Industrial Applications for Green Chemistry; American Physical Society: Washington, DC, 2002; Vol. 818. alternation apparent even in the disordered structure of IL fluid phases, and the exponential screening of all charge perturbations. ${ }^{5}$ The structural modulation at the mesoscopic scale ${ }^{6}$ displayed by several ILs might also be seen as being part of the same general picture, since it arises from the competition of short-range dispersion forces driving the aggregation of the cation tails, and of long-range Coulomb interactions, preventing the macroscopic separation of charge.

A further example of the general and fundamental aspects of Coulomb fluids is represented by the association of ions into neutral pairs ${ }^{7,8}$ or larger aggregates, taking place in systems of low charge density. ${ }^{9}$ This process can be seen either as a transformation of the microscopic structure and bonding from ionic to dipolar or, equivalently, as a change of the ion dynamics that reduces the transport of charge leaving nearly unaffected the diffusional motion of neutral (mass) currents. This second point of view is perhaps the most relevant one for experiments, because the apparent manifestation of ion association is a progressive violation of the Nernst-Einstein relation ${ }^{10}$ between the ionic conductivity and the diffusion coefficients of cations and anions $\left(D_{+}, D_{-}\right.$, respectively).

(3) Hansen, J. P.; McDonald, I. R. Theory of Simple Liquids; Academic Press: London, 1986

(4) March, N. H.; Tosi, M. P. Coulomb Liquids; Academic Press: New York, 1984.

(5) Stillinger, F. H.; Lovett, R. J. Chem. Phys. 1968, 49, 1991-1994.

(6) (a) Wang, Y.; Voth, G. A. J. Am. Chem. Soc. 2005, 127, 1219212193. (b) Canongia Lopes, J. N. A.; Pádua, A. A. H. J. Phys. Chem. B 2006, 110, 3330-3335.

(7) Bjerrum, N. K. Dan. Vidensk. Selsk. Mat.-Fys. Medd. 1926, $1,1$.

(8) Marcus, Y.; Hefter, G. Chem. Rev. 2006, 106, 4585-4621.

(9) Weingärtner, H.; Schröer, W. Adv. Chem. Phys. 2001, 116, 1-67.

(10) Atkins, P.; de Paula, J. Physical Chemistry, 7th ed.; W. H. Freeman \& Company: New York, 2002; p 775. 
The general relevance of ion association for Coulomb fluids is emphasized by the role that it plays in the phase diagram of prototypical Coulomb systems such as molten alkali halides, which in the vicinity of their liquid-vapor critical point undergo a transformation from the ionic liquid state to a fluid made of neutral ion pairs, ${ }^{9}$ interacting among themselves via strong dipolar forces. This change, which is believed to be a sharp but nevertheless continuous shift of chemical equilibria and not a genuine phase transition, greatly affects properties like ionic conductivity, viscosity, diffusion, and even optical absorption. It results from the competition between the energy cost of dissociating the molecular ion pair and the energy gain of sharing several equivalent nearest neighbors of opposite charges. Elementary considerations show that the ionic liquid state prevails at high density, whereas low density stabilizes the molecular phase. ${ }^{11}$

The small size and tight packing of traditional molten salts imply that the molecular to ionic transition takes place at very high temperature and pressure. ${ }^{12,13}$ Dissolving salts into water greatly decreases the strength of the Coulomb interactions and promotes the dissociation of ion pairs. This brings the transition temperature within an easily accessible range, but at the same time, it moves the corresponding ion density (or, equivalently, concentration) to very low values, ${ }^{14}$ making an investigation of the transformation using standard experimental techniques difficult. Moreover, it introduces the additional complication of the ions' interaction with a third species, that is, water, whose complexity is proverbial, and whose intermolecular interactions are also long ranged.

ILs provide an appealing new path to bring the molecular to ionic transformation in the foreground of easily accessible conditions that, in addition, can be fine-tuned by exploiting the wide variety of ILs now routinely synthesized and characterized. ${ }^{15}$ Moreover, several ILs are soluble in organic solvents, thus providing a simple way to vary the IL density over a wide range without incurring into the complexity and chemical activity of IL/water solutions. Perhaps more importantly, organic solvents of low dielectric constant do not significantly reduce the stability of neutral ion pairs, and the transition from ionic to dipolar bonding takes place at fairly high IL concentrations.

Recent measurements of the molar conductivity of IL solutions in neutral organic solvents ${ }^{16,17}$ display a conductivity maximum at IL concentrations of about $10 \%$, which is highly suggestive of an underlying molecular to ionic transformation.

(11) The ion pairs in the vapour phase of charged systems dissociate again into free ions at densities (or concentrations, in electrolyte solutions) much lower than those considered here.

(12) The critical point of $\mathrm{NaCl}$ is estimated to be at $T_{\mathrm{c}}=3300 \mathrm{~K}, \rho_{\mathrm{c}}=$ $0.18 \mathrm{~g} / \mathrm{cm}^{3}$, see: (a) Kirshenbaum, A. D.; Cahill, J. A.; McGonigal, P. J.; Grosse, A. V. J. Inorg. Nucl. Chem. 1962, 24, 1287-1296. (b) Guissani, Y.; Guillot, B. J. Chem. Phys. 1994, 101, 490-509.

(13) Grantham, L. F.; Yosim, S. J. J. Chem. Phys. 1966, 45, 1192-1198.

(14) (a) Pitzer, K. S. Acc. Chem. Res. 1990, 23, 333-338. (b) Fisher, M. E. Levin, Y. Phys. Rev. Lett. 1993, 71, 3826-3829.

(15) Together with their positive features, it might be useful to point out also the disadvantages of IL that, at variance from simple salts, are chemically stable only over a fairly limited temperature range. [bmim] $\left[\mathrm{PF}_{6}\right]$, for instance, thermally decomposes above $T \approx 349^{\circ} \mathrm{C}$; see: Huddleston, J.; Visser, A. E.; Reichert, W. M.; Willauer, H. D.; Broker, G. A.; Rogers, R. D. Green Chem. 2001, 3, 156-164.

(16) Jarosik, A.; Krajewski, S. R.; Lewandowski, A.; Radzimski, P. J. Mol. Liq. 2006, 123, 43-50.

(17) Comminges, C.; Barhdadi, R.; Laurent, M.; Troupel, M. J. Chem. Eng. Data 2006, 51, 680-685.

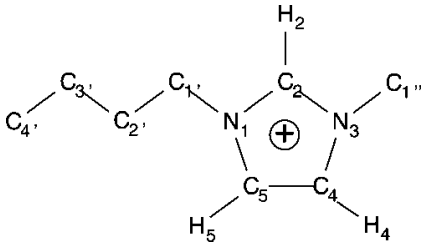

Figure 1. Schematic drawing of the structure of the 1-n-butyl-3-methylimidazolium cation $\left([\mathrm{bmim}]^{+}\right)$.

The result of other studies ${ }^{18}$ do not display such a maximum within the experimentally accessible range but highlight deviations from the ideal Nernst-Einstein behavior that point again to an incipient change in the system bonding and dynamics as a function of ion density. Although these anomalies are generally attributed to ion association, the microscopic origin of the conductivity maximum in IL has not been investigated in detail before.

In this work, we study the transformation of $\left[\mathrm{bmim}^{\mathrm{b}}\right]\left[\mathrm{PF}_{6}\right]$ (see Figure 1) in $[\mathrm{bmim}]\left[\mathrm{PF}_{6}\right] /$ naphthalene solutions from prototypical ionic liquid to a molecular fluid made of neutral ion pairs floating, interacting and condensing within liquid naphthalene. The transition takes place upon varying the concentration of $[\mathrm{bmim}]\left[\mathrm{PF}_{6}\right]$, that we measure by the mole fraction $x$ defined as $x=N_{[\mathrm{bmim}]\left[\mathrm{PF}_{6}\right]} /\left(N_{[\mathrm{bmim}]\left[\mathrm{PF}_{6}\right]}+N_{\mathrm{C}_{10}} \mathrm{H}_{8}\right)$, where $N_{[\mathrm{bmim}]\left[\mathrm{PF}_{6}\right]}$ and $N_{\mathrm{C}_{10}} \mathrm{H}_{8}$ are the number of [bmim] $\left[\mathrm{PF}_{6}\right]$ and naphthalene molecules, respectively. The thermodynamic and transport properties of $[\mathrm{bmim}]\left[\mathrm{PF}_{6}\right]$ and several of its mixtures are fairly well-known, but we decided to carry out an independent determination of density, viscosity and electric conductivity of $[\mathrm{bmim}]\left[\mathrm{PF}_{6}\right] /$ naphthalene solutions over the full concentration range $(0 \leq x \leq 1)$ to obtain a comprehensive and consistent set of data.

The experimental results show a clear maximum of the conductivity per ion at $x \approx 0.15$, whereas the viscosity displays a nearly monotonic increase from pure naphthalene to pure [bmim $]\left[\mathrm{PF}_{6}\right]$.

The computational analysis described in detail in the following sections confirms that the maximum is due to the transition from molecular to the ionic state taking place with increasing IL concentration, and allows us to identify several important features of this transition. We observe, in particular, that the neutral ion pairs present in the system at low $x$ condense into the linear filaments that represent the most characteristic structural motif of low-density dipolar systems. ${ }^{19}$

Once again we emphasize that the transformation in the bonding pattern described here for $[\mathrm{bmim}]\left[\mathrm{PF}_{6}\right] /$ naphthalene mixtures is a general feature of Coulomb systems, and it is likely to influence a wide range of IL properties, far beyond the specific example investigated in our study. Furthermore, the transition is expected to have important consequences for applications, since it affects the basic identity of expanded IL as ionic systems.

\section{Experimental Measurements}

The transition from an ionic liquid to a molecular vapor in Coulomb fluids is traditionally described as taking place with

(18) (a) Li, W.; Zhang, Z.; Han, B.; Hu, S.; Xie, Y.; Yang, G. J. Phys. Chem. B 2007, 111, 6452-6456. (b) Fraser, K. J.; Izgorodina, E. I.; Forsyth, M.; Scott, J. L.; MacFarlane, D. R. Chem. Commun. 2007, 381, 7-3819.

(19) Ganzenmüller, G.; Camp, P. J. J. Chem. Phys. 2007, 126, $191104-$ 1-191104-4. 
Table 1. Ratio of Ionic Liquid to Organic Concentration in the IL-Rich Phase of IL Solutions in Neutral Organic Species ${ }^{a}$

\begin{tabular}{|c|c|c|c|c|c|}
\hline System & Benzene & Naphthalene & Toluene & 1- Methylnaphthalene & Xylene \\
\hline $\mathrm{T}\left[{ }^{\circ} \mathrm{C}\right]$ & 25 & 80 & 25 & 25 & 25 \\
\hline$\left[\mathrm{C}_{1} \mathrm{mim}\right]\left[\mathrm{MeSO}_{4}\right]$ & - & 0.3 & 0.1 & 0.2 & - \\
\hline$\left[\mathrm{C}_{2} \mathrm{mim}\right]\left[\mathrm{EtSO}_{4}\right]$ & - & 1.2 & 0.2 & 0.4 & - \\
\hline$\left[\mathrm{C}_{4} \mathrm{mim}\right][\mathrm{Cl}]$ & - & 1.0 & - & - & - \\
\hline$\left[\mathrm{C}_{1} \mathrm{mim}\right]\left[\mathrm{PF}_{6}\right]$ & $2.0^{b}$ & 0.6 & - & 0.3 & - \\
\hline$\left[\mathrm{C}_{2} \mathrm{mim}\right]\left[\mathrm{PF}_{6}\right]$ & - & 1.4 & - & 0.3 & - \\
\hline$\left[\mathrm{C}_{4} \mathrm{mim}\right]\left[\mathrm{PF}_{6}\right]$ & $2.1^{b}$ & single phase & $0.8^{b}$ & - & $0.3^{b}$ \\
\hline$\left[\mathrm{C}_{6} \mathrm{mim}\right]\left[\mathrm{PF}_{6}\right]$ & $3.5^{b}$ & single phase & $1.6^{b}$ & - & $0.3^{b}$ \\
\hline$\left[\mathrm{C}_{10} \mathrm{mim}\right]\left[\mathrm{PF}_{6}\right]$ & 9.3 & single phase & 4.2 & - & - \\
\hline$\left[\mathrm{C}_{1} \mathrm{mim}\right]\left[\mathrm{NTf}_{2}\right]$ & 2.7 & single phase & 1.5 & - & - \\
\hline$\left[\mathrm{C}_{2} \mathrm{mim}\right]\left[\mathrm{NTf}_{2}\right]$ & $3.0^{b}$ & single phase & $1.7^{b}$ & 5.9 & - \\
\hline$\left[\mathrm{C}_{1} \mathrm{C}_{1} \operatorname{mim}\right]\left[\mathrm{NTf}_{2}\right]^{c}$ & 1.8 & 10.5 & - & - & - \\
\hline$\left[\mathrm{C}_{4} \mathrm{mim}\right]\left[\mathrm{NTf}_{2}\right]$ & $3.5^{b}$ & single phase & $2.8^{b}$ & 10.2 & $1.3^{b}$ \\
\hline$\left[\mathrm{C}_{6} \mathrm{mim}\right]\left[\mathrm{NTf}_{2}\right]$ & 5.3 & single phase & - & - & - \\
\hline$\left[\mathrm{C}_{10} \operatorname{mim}\right]\left[\mathrm{NTf}_{2}\right]$ & 12.7 & single phase & 8.5 & - & - \\
\hline
\end{tabular}

${ }^{a}$ Mixtures completely miscible at $1: 11$ ionic liquid:aromatic molar ratio are denoted as Single Phase. ${ }^{b}$ From reference 20. ${ }^{c}$ 1,2,3-Trimethylimidazolium bis $\{$ (trifluoromethyl)sulfonyl $\}$ imide.

increasing temperature, even though the transformation itself occurs because of the volume expansion due to the temperature rise. ${ }^{13}$ The limited thermal stability of ILs, however, does not allow the kind of temperature variations required to change the bonding character of the liquid, and the system volume, or, more precisely, the ion density will play the role of the basic independent variable in our study. Once again, large volume variations are not easily obtained in experiments, but mixing with a solvent might represent a suitable replacement for the volume expansion, provided the mixture is homogeneous. This is particularly justified when the solvent has a low dielectric constant, since in that case, at least to zero-order approximation, we can neglect the interaction of the IL ions with the neutral molecules.

As a first step of our investigation the miscibilities of a range of ILs with low dielectric constant aromatic solvents (benzene, naphthalene, toluene, xylene, and 1-methylnaphthalene) were screened using previously ${ }^{20}$ reported and new experimental data. ${ }^{21}$ The mixtures were studied at room temperature, except in the case of naphthalene where the temperature was $80^{\circ} \mathrm{C}$. The molar composition of the IL-rich phase of the saturated biphasic mixtures was determined by ${ }^{1} \mathrm{H}$ NMR. Monophases were inspected visually, and in a number of cases reached the maximum mole ratio $(11: 1)$ of aromatic:IL investigated. The results are reported in Table 1. It should be noted that, for the most diluted samples, microsegregation and subtle changes not affecting the color or the macroscopic system morphology might escape identification. This is in fact a real possibility, taking into account the remarkable complexity displayed by the phase diagram of IL/neutral organic solvent mixtures. ${ }^{22,23}$ Nevertheless, our data are consistent with previously published miscibility data for IL/aromatic mixtures ${ }^{20}$ and agree with a high accuracy phase diagram ${ }^{24}$ for $[\mathrm{dmim}]\left[\mathrm{PF}_{6}\right] /$ benzene. ${ }^{25}$ The previous experiments allowed to identify the combinations of ILs and

(20) Holbrey, J. D.; Reichert, W. M.; Nieuwenhuyzen, M.; Sheppard, O.; Hardacre, C.; Rogers, R. D. Chem. Commun. 2003, 476-477.

(21) All of the ILs were prepared by alkylation of the corresponding imidazole bases with bromoalkanes followed by anion metathesis with potassium hexafluorophosphate, or lithium bis\{(trifluoromethyl)sulfonyl \}imide, respectively using literature methods: (a) Gordon, C. M.; Holbrey, J. D.; Kennedy, A.; Seddon, K. R. J. Mater. Chem., 1998, 8, 2627-2636. (b) Bonhote, P.; Dias, A.-P.; Armand, M.; Papageorgiou, N.; Kalyanasundaram, K.; Grätzel, M. Inorg. Chem. 1996, 35, 1168 1178.

(22) Friedman, H. L. J. Phys. Chem. 1962, 66, 1595-1600.

(23) Lachwa, J.; Szydlowski, J.; Najdanovic-Visak, V.; Rebelo, L. P. N.; Seddon, K. R.; Nunes da Ponte, M.; Esperanca, J. M. S. S.; Guedes, H. J. R. J. Am. Chem. Soc. 2005, 127, 6542-6543.

(24) Lachwa, J.; Bento, I.; Duarte, M. T.; Canongia Lopes, J. N.; Rebelo, L. P. N. Chem Commun 2006, 2445-2447.

(25) $[\mathrm{dmim}]^{+}$is the 1,3-dimethylimidazolium cation.

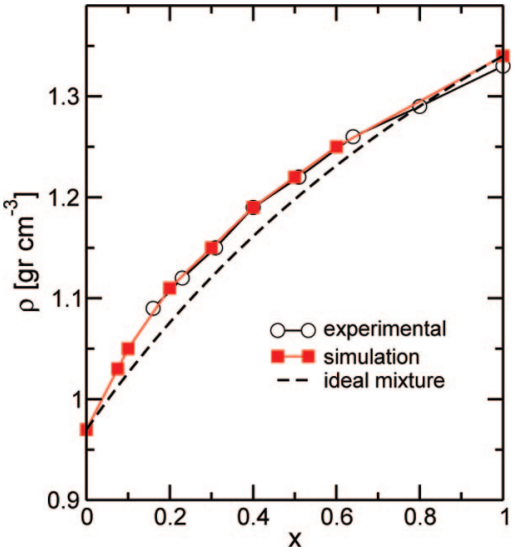

Figure 2. Comparison of measured and computed density of $[\mathrm{bmim}]\left[\mathrm{PF}_{6}\right] /$ naphthalene mixtures as a function of composition. The simulation results have been obtained by MD in the NPT ensemble at $T=350 \mathrm{~K}, P=1 \mathrm{~atm}$. Experimental measurements have been carried out at the same pressure and $T=353 \mathrm{~K}$. The density of the corresponding ideal mixture as a function of composition is reported to provide a natural density scale.

aromatic solvents leading to a homogeneous mixture over the widest possible concentration range. The high miscibility of naphthalene with many of the ILs screened clearly points to this compound as the most suitable solvent for our investigation. The choice of [bmim] $\left[\mathrm{PF}_{6}\right]$, on the other hand, has been made because it is a prototypical IL, widely available, and easily prepared at the level of purity required for a precise determination of transport properties.

$[\mathrm{bmim}]\left[\mathrm{PF}_{6}\right]$ was prepared from $[\mathrm{bmim}] \mathrm{Br}$ following the method of Friberg et al. ${ }^{26}$ Prior to use the ionic liquid was dried under vacuum at $80{ }^{\circ} \mathrm{C}$ and was stored in a glovebox. Water content was determined by the Karl Fischer analysis to be $0.36 \mathrm{~mol} \%$. Naphthalene was used as received from Aldrich. Ten samples of [bmim $]\left[\mathrm{PF}_{6}\right] /$ naphthalene solutions were prepared in a glovebox containing mole fractions of ionic liquid with respect to naphthalene of 1.0 (pure IL), 0.80, 0.64, 0.51, 0.40, 0.31, 0.23, 0.16, 0.10, and 0.05 . No pure naphthalene sample was investigated because this compound is solid at the temperature of our transport measurements ( $T=80{ }^{\circ} \mathrm{C}$ ), consistent with the fact that the melting temperature of naphthalene $\left(T_{\mathrm{m}}=80.5^{\circ} \mathrm{C}\right)$ is slightly higher.

Electrical conductivity was measured by using a Jenway Conductivity Meter 4320, with a three point calibration using a standard dilution method. The viscosity and density of each sample was also measured using a Brookfield DV-II viscometer and an Anton Paar DMA 4500 densitometer, respectively. All measurements were performed at $80{ }^{\circ} \mathrm{C}$ under argon.

Naphthalene and $[\mathrm{bmim}]\left[\mathrm{PF}_{6}\right]$ have similar densities, and the data for the mixtures join smoothly and monotonically the two extreme cases (see Figure 2). The $\rho(x)$ curve, however, apparently deviates from the analytic expression valid for ideal mixtures. The density data have been used to gauge the interatomic potential and are discussed in more detail in the Supporting Information.

Our results for the viscosity coefficient of mixtures as a function of composition are reported in Figure 3. Close to their melting point, ILs are usually fairly viscous fluids, and also in the present case, the viscosity of the solvent (naphthalene) is much less than that of [bmim $]\left[\mathrm{PF}_{6}\right]$. Disregarding the slight anomaly at intermediate composition $(x \approx 0.4-0.5)$, the measured viscosities display a nearly monotonic increase with increasing IL concentration from pure naphthalene to pure $[\mathrm{bmim}]\left[\mathrm{PF}_{6}\right]$.

The results for the electrical conductivity $\sigma$ are collected in Figure 4. The raw data appear to be even smoother and more regular than those for viscosity. Starting from a fairly high value for pure

(26) Friberg, S. E.; Yin, Q.; Pavel, F.; Mackay, R. A.; Holbrey, J. D.; Seddon, K. R.; Aikens, P. A. J. Dispersion Sci. Technol. 2000, 21, 185-198. 


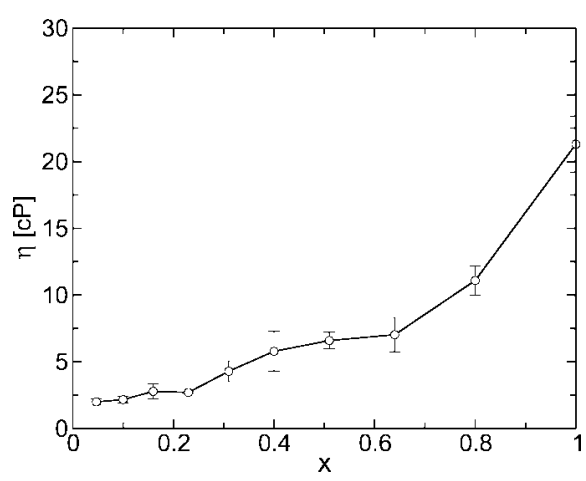

Figure 3. Viscosity coefficient $\eta$ of $[\mathrm{bmim}]\left[\mathrm{PF}_{6}\right] /$ naphthalene mixtures as a function of IL mole fraction $x$ measured at $T=353 \mathrm{~K}, P=1 \mathrm{~atm}$.

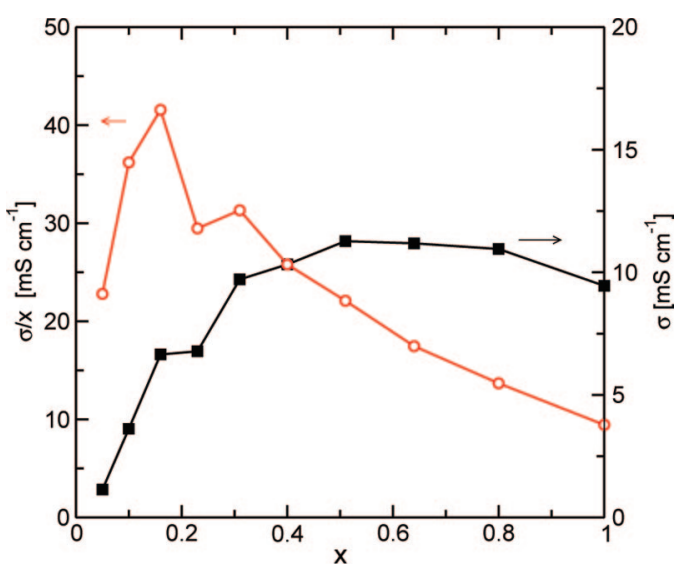

Figure 4. Right scale and $\mathbf{a}$ : ionic conductivity of $[\mathrm{bmim}]\left[\mathrm{PF}_{6}\right] /$ naphthalene mixtures as a function of IL mole fraction $x$ measured at $T=353 \mathrm{~K}, P=$ $1 \mathrm{~atm}$. Left scale and $\mathrm{O}$ : conductivity per ion $\sigma(x) / x$ (see ref 27).

[bmim] $]\left[\mathrm{PF}_{6}\right]$, conductivity increases slightly with decreasing IL content down to $x \approx 50 \%$, apparently because the related decrease of viscosity overcompensates the reduction of the carriers density. Further reduction of the IL concentration below $x=50 \%$ brings about a rapid drop of $\sigma$ obviously vanishing in the $x \rightarrow 0$ limit. However, once the effect of the decreasing carrier concentration is factored out by plotting the conductivity per ion $\sigma / x,{ }^{27}$ the experimental data show a different picture (see Figure 4) and display a clear conductivity maximum at $0.10 \leq x \leq 0.15$.

\section{Atomistic Simulation of $[\mathrm{bmim}]\left[\mathrm{PF}_{6}\right] /$ Naphthalene Mixtures}

The relation between the macroscopic properties and the atomistic picture has been investigated using computer simulation. This section contains a short outline of the model and simulation method and a detailed description of the simulation results. All simulations but one have been performed at $T=$ $350 \mathrm{~K}$, or $77{ }^{\circ} \mathrm{C}$, closely matching the temperature of the experimental measurements $\left(80^{\circ} \mathrm{C}\right)$. A single simulation has been performed at $T=400 \mathrm{~K}$ for a limited exploration of temperature effects.

A. Force Field Model. Molecular dynamics simulations of $[\mathrm{bmim}]\left[\mathrm{PF}_{6}\right] /$ naphthalene mixtures have been performed using an empirical, nonpolarizable force field, whose analytic form is given in ref 28. Several parametrizations of the force field to

(27) The conductivity per ion $\sigma / x$ is related to the molar conductivit by a simple transformation. The $x$-dependence of the two quantities is qualitatively the same. model either organic aromatic systems or IL are available ${ }^{28,29}$ and are generally successful in their respective domains. However, modeling mixtures of compounds with a significantly different intermolecular bonding character such as $[\mathrm{bmim}]\left[\mathrm{PF}_{6}\right]$ and benzene or naphthalene requires additional care, as already emphasized in the literature. ${ }^{30} \mathrm{We}$ verified that combining parameters for $[\mathrm{bmim}]\left[\mathrm{PF}_{6}\right]$ and for benzene or naphthalene from different sources ${ }^{31}$ admittedly an overly simplistic choice, easily leads to qualitatively wrong results for the mixtures, even when the two starting parametrizations are closely related. The stability range of homogeneous solutions, in particular, depends sensitively on the choice of the force field parameters and provides a useful diagnostic property to assess the overall quality of the model and to tune the cross interactions among different species.

The problematic description of mixing properties appears to be due to the uncertain attribution of cohesion to Coulomb and to dispersion forces and to the neglect of electronic polarization, which in reality provides a sizable contribution to the binding of ions to aromatic species (see also the discussion reported in the Supporting Information). Nevertheless, an extensive set of test computations has shown that combining the IL force field and parametrization from ref 38 for $\left[\mathrm{bmim}^{2}\left[\mathrm{PF}_{6}\right]\right.$ with the parameters for naphthalene from refs 29 and 32 provides a reliable model for the investigation of structural and dynamical properties of $[\mathrm{bmim}]\left[\mathrm{PF}_{6}\right] /$ naphthalene mixtures. In all cases, the nonbonded cross interactions among the different atom types defined by the force field model are obtained by ideal mixing rules. $^{31}$

The model, in particular, is able to reproduce the crystal structure of $[\mathrm{bmim}]\left[\mathrm{PF}_{6}\right]$, of naphthalene, as well as the complex clathrate structure of 1,3-methylimidazolium hexafluorophosphate/ 0.5 benzene crystals, ${ }^{20}$ whose bonding pattern is apparently closely related to that of $[\mathrm{bmim}]\left[\mathrm{PF}_{6}\right] /$ naphthalene mixtures. Moreover, it reproduces remarkably well the concentration dependence of the density over the entire $0 \leq x \leq 1$ range (see Figure 2). Visual inspection of samples equilibrated over long $\mathrm{MD}$ runs shows that $[\mathrm{bmim}]\left[\mathrm{PF}_{6}\right] /$ naphthalene mixtures are homogeneous down to a concentration $x \approx 0.2$. Homogeneity is less apparent at lower concentrations $(x \leq 0.1)$ but in this narrow composition range also the results of experiments become somewhat less certain. A more quantitative assessment of the homogeneity of low- $x$ mixtures is given below.

Additional details on the force field, and on the selection of the IL/naphthalene parametrization are collected in the Supporting Information.

B. Simulation Method. We consider neutral samples made of $N_{\text {[bmim][PF }}[\mathrm{bmim}]\left[\mathrm{PF}_{6}\right]$ ion pairs and $N_{\mathrm{C}_{10}} \mathrm{H}_{8}$ naphthalene molecules at concentration $x=N_{[\mathrm{bmim}]\left[\mathrm{PF}_{6}\right.} /\left(N_{[\mathrm{bmim}]\left[\mathrm{PF}_{6}\right]}+\right.$ $N_{\mathrm{C}_{10}} \mathrm{H}_{8}$ ), enclosed in a cubic simulation box periodically repeated in space. Simulations have been carried out using the DL_POLY package. ${ }^{39}$ A time step of $1 \mathrm{fs}$ guarantees a good conservation

(28) Canongia Lopes, J. N.; Dechamps, J.; Padua, A. A. H. J. Phys. Chem. $B$ 2004, 108, 2038-2047.

(29) Jorgensen, W. L.; Maxwell, D. S.; Tirado-Rives, J. J. Am. Chem. Soc. 1996, 118, 11225-11236.

(30) (a) Harper, J. B.; Lynden-Bell, R. M. Mol. Phys. 2004, 102, 85-94. (b) Hanke, C. G.; Johansson, A.; Harper, J. B.; Lynden-Bell, R. M. Chem. Phys. Lett. 2003, 374, 85-90.

(31) The cross interactions are determined by simple mixing rules $\left(\varepsilon_{i j}=\right.$ $\left(\varepsilon_{i} \varepsilon_{j}\right)^{1 / 2}$ and $\left.\sigma_{i j}=\left(\sigma_{i} \sigma_{j}\right)^{1 / 2}\right)$ without further tuning.

(32) The atomic charges for naphthalene, not listed in ref 29 , have been determined by fitting the molecular electrostatic potential computed by Møller-Plesset perturbation theory of second order (MP2). 
Table 2. IL Concentration $x$, Number of IL Molecules $N_{\mathrm{IL}}$, of Naphthalene Molecules $N_{\mathrm{C}_{10}} \mathrm{H}_{8}$, Length of the Equilibration (teq and Statistics $\left(t_{\text {stat }}\right)$ Runs Performed in Our Study ${ }^{a}$

\begin{tabular}{clrccc}
\hline sample & \multicolumn{1}{c}{$x$} & $N_{\mathrm{LL}}$ & $N_{\mathrm{C}_{10}} \mathrm{H}_{8}$ & $t_{\text {eq }}(\mathrm{ns})$ & $t_{\text {stat }}(\mathrm{ns})$ \\
\hline 1 & 0.075 & 360 & 29 & 1.25 & 6 \\
2 & 0.075 & 720 & 58 & 1.25 & 6 \\
3 & 0.1 & 360 & 40 & 1.25 & 11 \\
4 & 0.2 & 320 & 80 & 1.25 & 4 \\
5 & 0.29 & 120 & 50 & 1.25 & 4 \\
6 & 0.4 & 120 & 80 & 1.25 & 5 \\
7 & 0.5 & 80 & 80 & 1.25 & 4 \\
8 & 0.6 & 120 & 80 & 1.25 & 6
\end{tabular}

${ }^{a}$ Simulations have been performed at $T=350 \mathrm{~K}$ at $P=1 \mathrm{~atm}$. Additional simulations have been performed for sample 4 at $T=400 \mathrm{~K}$, with $t_{\mathrm{eq}}=2 \mathrm{~ns}$, and $t_{\mathrm{stat}}=2 \mathrm{~ns}$.

of the constant of motion appropriate for the selected ensemble. The electrostatic energy is computed using the Ewald technique.

Each sample has been equilibrated for at least $1 \mathrm{~ns}$, during simulation runs performed in the NPT ensemble keeping fixed the cubic shape of the simulation cell. The NPT ensemble conditions are enforced using the Nosé-Hoover extended Lagrangian formulation both for temperature and for pressure, ${ }^{40}$ with relaxation times of $0.1 \mathrm{ps}$ for temperature and $0.2 \mathrm{ps}$ for pressure.

Statistics has been accumulated over runs lasting at least 4 ns. Longer runs have been carried out at the lowest concentrations to compensate for the low number of ions and large fluctuations of these samples. Production runs have been carried out in the NVE ensemble, at a volume equal to the average value obtained during the last $20 \%$ of the NPT stage. The change of ensemble from the equilibration runs (NPT) to the production stage (NVE) has been made to prevent artifacts in the real time dynamics due to the fictitious volume and time fluctuations introduced by the NPT algorithm.

The analysis of trajectories and the computation of average quantities have been carried out using methods similar to those detailed in ref 41 . Further information on the computation of dynamical coefficients and of the associated error bars are given in Sec. II of the Supporting Information.

\section{Simulation Results}

A summary of the simulations performed in our study is given in Table 2, listing the composition and size of all the simulated samples, together with the length of the equilibration and production runs carried out for each of them. Samples of the two extreme compositions, that is, liquid $\left[\mathrm{bmim}^{\mathrm{m}}\right]\left[\mathrm{PF}_{6}\right]$ and liquid naphthalene, have been simulated as well, to provide a further test of the force field and to collect data for the computation of excess quantities. Our results for liquid $\left[\mathrm{bmim}^{-}\left[\mathrm{PF}_{6}\right]\right.$ agree well with those of previous simulations. ${ }^{33}$ Only a few computational studies have been reported for naphthalene, ${ }^{34}$ and unfortunately, their results are not directly comparable to ours because of essential differences in the underlying model. The results of our simulation of liquid naphthalene will be reported elsewhere.

The first qualitative analysis of the mixture properties is obtained by inspecting snapshots of the simulated systems (see Figure 5). As already mentioned in Sec. 3.1, the samples of composition $0.2 \leq x \leq 1$ are apparently homogeneous.

(33) (a) Margulis, C. J.; Stern, H. A.; Berne, B.J. J. Phys. Chem. B 2002 , 106, 12017-12021. (b) Morrow, T. I.; Maginn, E. J. J. Phys. Chem. B 2002, 106, 12807-12813.

(34) Sediawan, W. B.; Gupta, S.; McLaughlin, E. J. Chem. Phys. 1989 90, 1888-1900.
Moreover, ions do not display any tendency to form closely associated neutral pairs. Instead, each ion appears to be equally shared among several neighbors of opposite charge, consistent with the basic idea of ionic liquids. ${ }^{35}$

Snapshots of the $x=0.075$ and $x=0.1$ samples, however, reveal a different picture. In these cases, the $[\mathrm{bmim}]^{+}$and $\left[\mathrm{PF}_{6}\right]^{-}$ ions tend to form globular aggregates of nanometric dimensions, joined by elongated structures (filaments) also made of [bmim $]^{+}$ and $\left[\mathrm{PF}_{6}\right]^{-}$ions. $^{42}$ Naphthalene molecules cluster in the voids left in between the aggregates and filaments. The elongated IL structures are very thin, consisting in fact of 1D chains of alternating cations and anions. The $[\mathrm{bmim}]\left[\mathrm{PF}_{6}\right]$ aggregates can be divided into two classes. A few of them apparently result from the coiling of ion chains, whose structure is still recognizable. Most of the aggregates, however, look like small droplets of the dense IL and, therefore, appear to be ionic.

We emphasize that in all the snapshots analyzed (including the larger sample at $x=0.075$ ), aggregates and chains stretch across the entire simulation cell, and an isolated chain segment or ring was never found. The absence of open chains might be due to the high potential energy of the bare ionic terminations, while rings might be penalised by the high entropy cost of closing a chain on itself. Thus, the combination of aggregates and filaments percolating across the entire system might indeed represent the most convenient configuration at low IL content. The mass ratio of filaments and blobs depends on composition, shifting toward a higher proportion of filaments with decreasing IL concentration.

Of the three species present in our system, that is $[\mathrm{bmim}]^{+}$, $\left[\mathrm{PF}_{6}\right]^{-}$, and naphthalene, only the first one gives rise to different conformers at room temperature, corresponding to different rotational isomers of the butyl hydrocarbon chain. Our simulations reveal systematic variations in the $[\mathrm{bmim}]^{+}$conformation as a function of concentration, which become slightly more marked in the $0.1 \leq x \leq 0.2$ range. However, these changes are quantitatively too small to allow a detailed investigation of their origin or effect, and therefore they will not be discussed again in what follows.

The complex structure seen in our samples points to the formation of a mesophase in mixtures of low IL content. The same data, however, could point to a different scenario. The complex structures seen in our simulation could in principle represent a transient stage toward the macroscopic phase separation of two components out of the original homogeneous solution, whose completion might have been prevented by the small sample size and by the short observation time. ${ }^{43}$ Simulation alone cannot provide a fully unambiguous identification of the correct picture, but the prediction of a mesophase formation at low $x$ is supported by the results of several computational tests. First of all, we compared the structures of Sample 1 and Sample 2 of Table 2, whose compositions are the same $(x=0.075)$ and differ only because the size of Sample 2 is twice that of Sample 1. Upon equilibration, the two samples appear to be completely equivalent, displaying structures that are geometrically indistinguishable from each other. Moreover, despite the relatively long simulation times and the good mobility of ions and molecules, we do not observe any systematic growth of the different structural components (chains, blobs, naphthalene clusters) and, even less, a clear phase segregation with the formation of a simple interface. A more

(35) The result might be different in IL with a long hydrocarbon tail, since in that case there is the formation of a mesophase reference. Gorden, C. Am.; Holbrey, J. D.; Kennedy, A. R.; Seddon, K. R. J. Mater. Chem. 1998, 8, 2627-2636. 
(a)

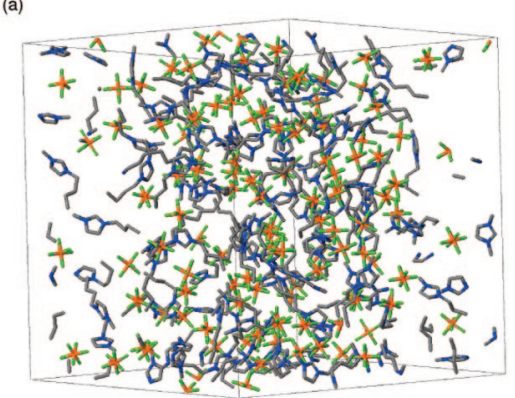

(c)

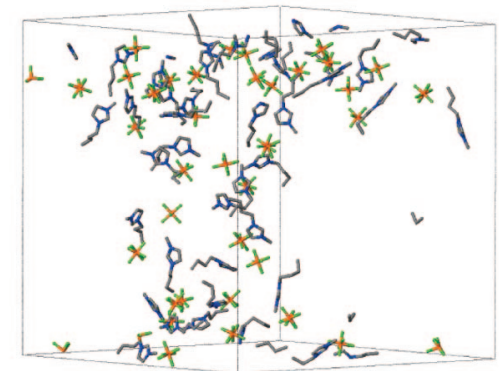

(b)

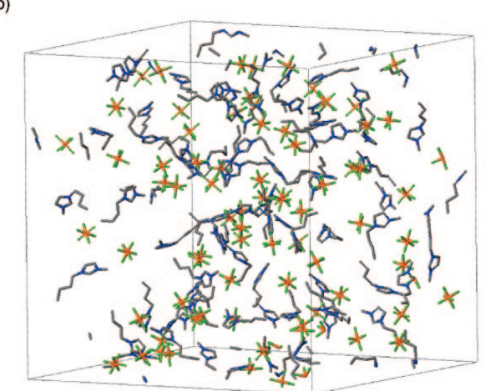

(d)

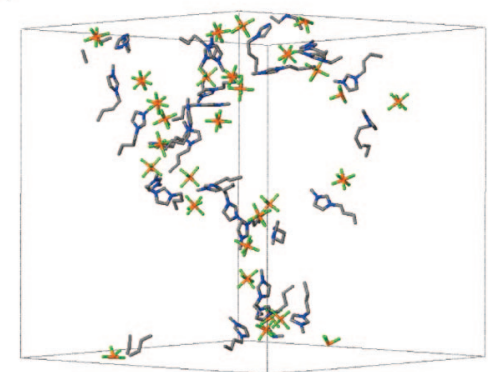

Figure 5. Snapshots of simulated samples at mole fractions of: (a) $x=0.60 ;$ (b) $x=0.20 ;$ (c) $x=0.10$; (d) $x=0.075$. $T=350 \mathrm{~K}$ and $P=1$ atm. Files with atomic positions for $x=0.1$ and $x=0.075$ are provided as Supporting Information.

quantitative analysis, based on the computation of the structure factor, is discussed below.

The most convincing evidence supporting a mesophase formation is provided by the results of a computer experiment performed on a system larger and more dilute (i.e., lower $x$ ) than either Sample 1 and Sample 2. The simulation of this third low- $x$ sample started from a phase-separated configuration, with all the $[\mathrm{bmim}]^{+}$and $\left[\mathrm{PF}_{6}\right]^{-}$grouped into a globular droplet of $2.6 \mathrm{~nm}$ diameter, centered at the origin, and surrounded by a large $\mathrm{C}_{10} \mathrm{H}_{8}$ buffer. After a short equilibration stage lasting 500 ps and including constraints stabilizing the phase-separated configuration, the simulation was continued without constraints in the NPT ensemble for a total of 5 ns. During this time, we observe the progressive dissolution of the original droplet, giving rise to a structure qualitatively indistinguishable from that found in the main simulation sequence. This observation suggests that the peculiar geometries described above are not an artifact caused by the long delay required to nucleate the phase separation during a MD simulation. One could still argue that the segregation is prevented by the small size of the simulated sample, that excludes the formation of even the smallest stable IL nucleus embedded into naphthalene. The comparison of Sample 1 and Sample 2 described in the previous paragraph, however, suggests that the size dependence of the simulation results is fairly mild. Moreover, the interfacial tension, that decides the minimum size of the stable seed, appears to be fairly low, as suggested by the high miscibility of the solutions, and by the wide contact of IL and naphthalene in well equilibrated samples at all concentrations.

The strict alternation of cations and anions and especially the formation of filaments suggest that a sizable fraction of the ions in low- $x$ samples are associated into neutral pairs, that bind to each other via dipolar forces. ${ }^{37}$ In other words, the simple

(36) See page 451 of ref 3.

(37) (a) de Gennes, P. G.; Pincus, P. A. Phys. Kondens. Mater. 1970, 11, 189. (b) Weis, J. J.; Levesque, D. Phys. Rev. Lett. 1993, 71, 27292732 . visualization of the simulated samples of $\left[\mathrm{bmim}^{\mathrm{b}}\right]\left[\mathrm{PF}_{6}\right]$ mixtures already points to a simultaneous change of geometry and bonding taking place between $x=0.1$ and $x=0.2$. The sizable fraction of ions that condense into dipolar chains especially at low IL concentrations motivates our (admittedly loose) usage of the term transition to characterize the shift of equilibrium between the ionic and molecular forms of [bmim] $\left[\mathrm{PF}_{6}\right]$.

The most apparent experimental evidence that something remarkable is taking place in $[\mathrm{bmim}]\left[\mathrm{PF}_{6}\right] /$ naphthalene mixtures is provided by the peak in the conductivity per ion seen in Figure 4 at low IL concentrations. Therefore, we computed the ionic conductivity of the simulated samples to verify that our model reproduces the experimental behavior. As expected and as discussed in several papers, ${ }^{44}$ the determination of the conductivity is fairly challenging for all ionic systems (see also the discussion reported in the Supporting Information), but for dilute IL solutions in neutral solvents it is at the limit of what can be computed in practice. Nevertheless, the simulation results shown in Figure 6 compare fairly well with the experimental data, and the agreement is particularly good at high IL concentrations. Sizable deviations in the absolute value of the conductivity appear at $x \leq 0.5$, probably due to the approximations made by the force field model. However, the qualitative trends of the

(38) Bhargava, B. L.; Balasubramanian, S. J. Chem. Phys. 2007, 127, 114510-114516.

(39) Smith, W.; Leslie, M.; Forester, T. R.; DL-POLY, Version 2.14; Daresbury: Warrington, UK, 2003.

(40) Melchionna, S.; Ciccotti, G.; Holian, B. L. Mol. Phys. 1993, 78, $533-$ 544.

(41) Salanne, M.; Simon, Ch.; Turq, P.; Madden, P. A. J. Chem. Phys. B 2007, 111, 4678-4684.

(42) The structures described in the text are not easy to identify in the printed Figure 5. However, they are clearly seen by visualising configurations even with simple graphic tools that allow to rotate the simulated samples. For this reason, the atomic coordinates of selected configurations at $x=0.075$ and $x=0.1$ are added to the Supporting Information.

(43) Sieffert, N.; Wipff, G. J. Phys. Chem. B 2006, 110, 13076-13085.

(44) See, for instance: Ciccotti, G.; Jacucci, G.; McDonald, I. R. Phys. Rev. A 1976, 13, 426-436. 


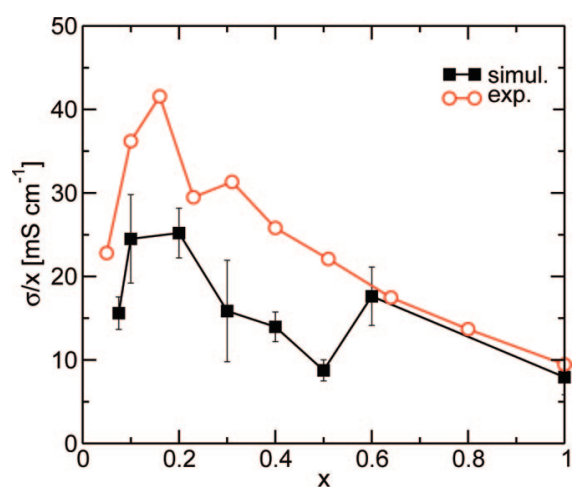

Figure 6. Comparison of measured and computed conductivity per ion $\sigma(x) / x$ as a function of composition. Experimental measurements at $T=$ $353 \mathrm{~K}$, simulation results obtained for $T=350 \mathrm{~K}$.

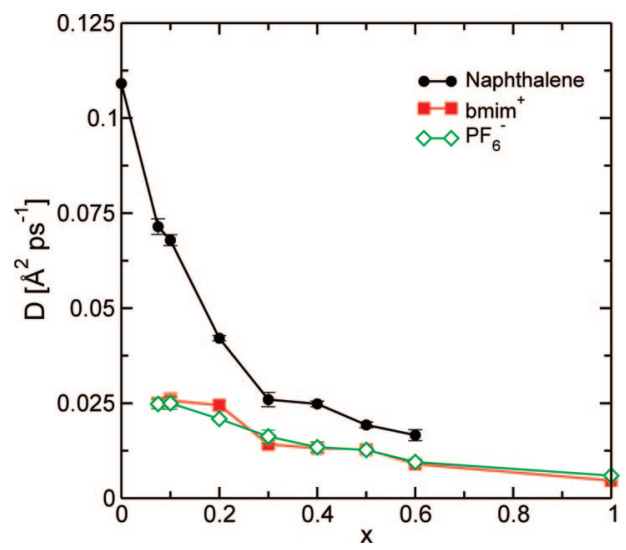

Figure 7. Computed diffusion coefficients of $[\mathrm{bmim}]^{+},\left[\mathrm{PF}_{6}\right]^{-}$, and naphthalene at $T=350 \mathrm{~K}$ and $P=1 \mathrm{~atm}$.

experimental data are reproduced by the simulation results, and the peak at low IL concentration is the most remarkable feature of the $\sigma(x) / x$ versus $x$ curve both in the experiments and in the simulation.

A first link between the conductivity data and the microscopic dynamics is established by computing the diffusion coefficient for all the species in the mixture. The results are reported in Figure 7. No experimental data are available for a comparison, but the low error bar ${ }^{45}$ on the computed $D_{+}$and $D_{-}$enhance our confidence on the internal consistency of the diffusion coefficients estimated during our MD simulations.

First of all naphthalene is always the most mobile component, as could have been expected since the viscosity of liquid $\mathrm{C}_{10} \mathrm{H}_{8}$ is much less than that of $[\mathrm{bmim}]\left[\mathrm{PF}_{6}\right]$. Despite the marked asymmetry of anions and cations, their diffusion coefficient is very similar, as it is often the case for electrolyte species whose motion is highly correlated. ${ }^{46}$ Starting from a relatively low value at $x=1.0$, the diffusion coefficients of cations and anions ( $D_{+}$and $D_{-}$, respectively) increase slowly but monotonically with decreasing $x$ down to $x \approx 0.2$, probably because of the corresponding decrease of the system viscosity. However, both $D_{+}$and $D_{-}$display a plateau for $0 \leq x \leq 0.2$, in sharp contrast with the remarkable rise of $D_{\mathrm{C}_{10} \mathrm{H}_{8}}$ over the same concentration

(45) The estimated relative error on $D_{+}, D_{-}$is $10 \%$, see the Supporting Information.

(46) For instance, simulation data for alkali halide salts show that the diffusion coefficient of anion and cation is similar even for ions whose size and mass are as different as those of $\mathrm{Na}^{+}$and $\mathrm{I}^{-}$. See Table 2 in ref 44 .

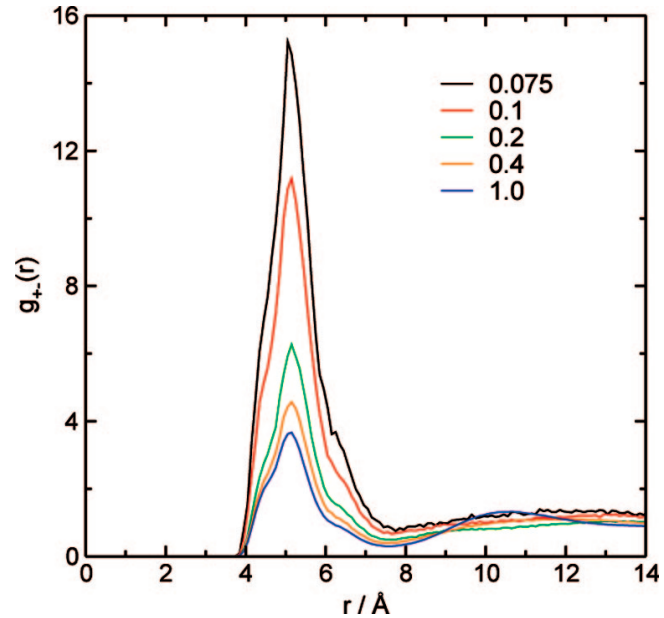

Figure 8. Radial distribution function for the geometric centres of [bmim $]^{+}$ and $\left[\mathrm{PF}_{6}\right]^{-}$(see text) computed by simulation at $T=350 \mathrm{~K}$ and $P=1 \mathrm{~atm}$ for different IL mole fractions $x$.

range, a behavior that in electrolytic solutions is often attributed to the formation of molecular and ionic complexes. At the very least, these results confirm the relevance of the conductivity anomaly, and point to a change in the aggregation state of the ions in solutions of low IL content.

The characterization of the local average structure of molecular liquids via their pair distribution functions is obviously far more challenging and less direct than in the case of atomic liquids. The simplicity of this analysis is to some extent restored by considering the radial distribution functions computed with respect to the geometric center of each ionic or molecular unit. In the case of $\left[\mathrm{PF}_{6}\right]^{-}$and naphthalene, this reference point coincides with their center of mass, whereas in the case of $[\mathrm{bmim}]^{+}$, it is the center of the imidazolium ring. The results are shown in Figure 8. At first sight these functions seem to provide little new information apart from displaying a remarkable alternation of anions and cations, emphasizing once again the role of Coulomb interactions down to the lowest IL concentrations. A closer look, however, shows that the $x$ dependence of the peak of the cation-anion radial distribution function, $g_{+-}$, is fairly different at low and at high IL concentrations. Starting from the pure IL side, the height of the $g_{+-}$peak changes only slightly with decreasing IL concentration down to $x=0.4$. The rate of change of the $g_{+-}$peak increases somewhat between $x=0.4$ and $x=0.2$ and finally displays a major enhancement below this concentration. The radial location of the first peak of all radial distribution functions is nearly independent of IL concentration in the mixture.

Comparing the radial distribution functions as defined above for the ionic species $[\mathrm{bmim}]^{+}$and $\left[\mathrm{PF}_{6}\right]^{-}$with those of simple (inorganic) ionic liquids might be misleading. Nevertheless, we remark that the peak of $g_{+-}$at $r=5 \AA$ is an order of magnitude higher than in the case of simple ionic liquids (such as $\mathrm{NaCl}$ ) near their triple point, ${ }^{3,4}$ and this observation alone already provides a strong evidence for ion pairing. This conclusion is strengthened by the $x$ dependence of the cation-cation and anion-anion radial distribution functions (see Figure 9). In this case, $g_{++}$and $g_{--}$are nearly independent of $x$ from $x=1.0$ down to $x=0.2$. The change of behavior at lower concentration, revealed by a drastic growth of the first peak of $g_{++}$and $g_{--}$ with decreasing $x$, is even more apparent than in the case of the $g_{+-}$radial correlation function. Moreover, the enhancement 


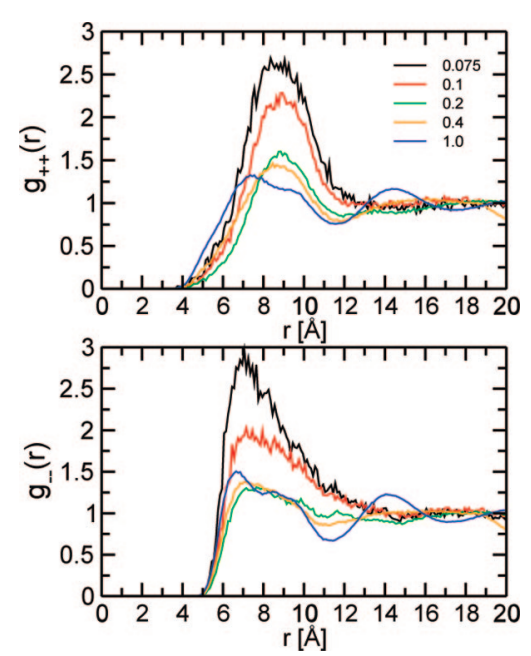

Figure 9. Composition dependence of the radial distribution functions $g_{++}$ and $g_{--}$for the geometric centres $[\mathrm{bmim}]^{+}$and $\left[\mathrm{PF}_{6}\right]^{-}$(see text) computed by simulation at $T=350 \mathrm{~K}$ and $P=1 \mathrm{~atm}$.

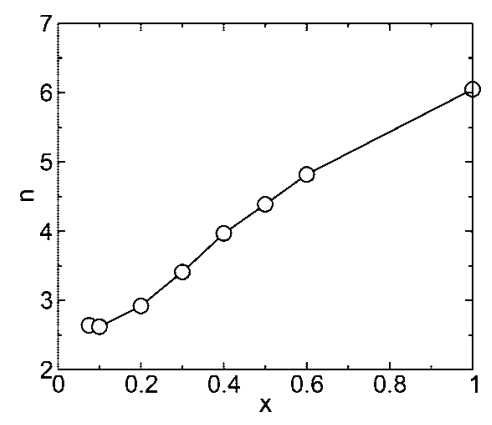

Figure 10. Anion-anion coordination number $n$ computed by MD simulation using a spherical cutoff radius $R_{\mathrm{c}}=8 \AA$. Distances are computed withe respect to the geometric centre of $[\mathrm{bmim}]^{+}$and $\left[\mathrm{PF}_{6}\right]^{-}$.

of correlation between like charges in mixtures of progressively vanishing ionic concentration is an obvious sign of clustering.

For completeness we mention that we do not observe any significant variation in the structure of naphthalene over the 0 $\leq x \leq 0.5$ composition range, confirming that the solvent behaves like an inert filling that allows the progressive expansion of the IL component.

The formation of neutral ion pairs at low IL concentration is confirmed by the computation of the coordination number $\langle n\rangle$ for the ions, defined as the average number of anions within a distance $R_{\mathrm{c}}$ from a central cation. For a 1:1 electrolyte such as [bmim] $\left[\mathrm{PF}_{6}\right]$ the result is the same if the role of anions and cations is reversed in the definition given above. As it is usually done, we set $R_{\mathrm{c}}$ to the radius of the first minimum in $g_{+-}$, that is, $R_{\mathrm{c}}=8 \AA$. The results are reported in Figure 10 . The coordination of pure $[\mathrm{bmim}]\left[\mathrm{PF}_{6}\right]$ turns out to be 6 , reminiscent of the 6-fold coordination of spherical and unpolarisable anions and cations in the $\mathrm{NaCl}$ structure. The composition dependence of $\langle n\rangle$ is slightly sublinear from $x=0.3$ to $x=1.0(\langle n\rangle \approx 1+$ $\left.5 x^{0.6}, 0.3 \leq x \leq 1\right)$. This behavior, together with the slow but monotonic rise of the $g_{+-}$peak with decreasing $x$, suggests that the rarefaction of ions is partly compensated by the enhanced stability of the first coordination shell. The coordination number remains nearly constant for $0 \leq x \leq 0.2$ at a value that is only slightly higher than two, consistently with the formation of linear chains of ions in equilibrium with nanometric ionic droplets.

Further insight into the nature and bonding of the IL filaments is obtained by the following analysis of the angles among triplets
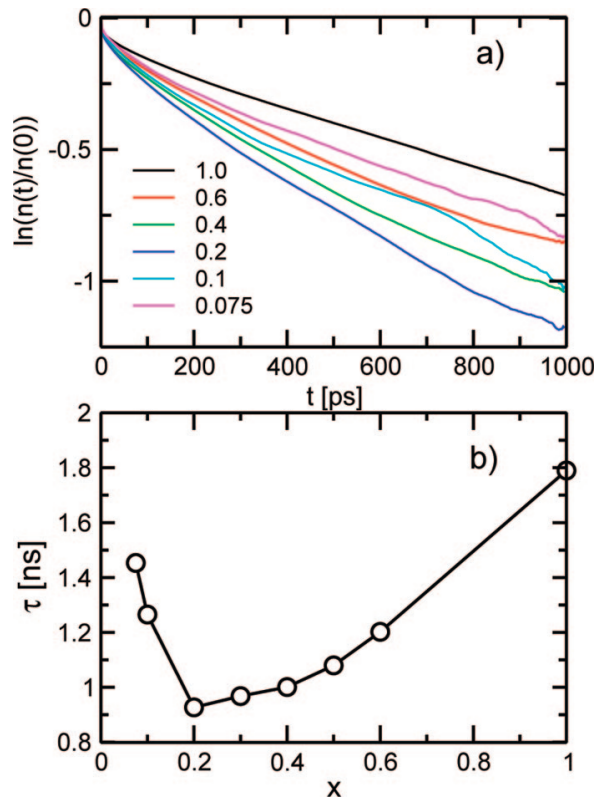

Figure 11. (a) Persistence index $n(t)$ as a function of $t$ for $[\mathrm{bmim}]\left[\mathrm{PF}_{6}\right]$ mixtures at various concentrations. $n(t)$ is the number of ion pairs (per ion) belonging to the first coordination shell $\left(R_{\mathrm{c}}=8 \AA\right)$ at $t=0$ and at a generic time $t$. (b) Time constant of an exponential fit for $n(t)$.

of neighboring ions. For each ion $i$ we identified all nearest neighbors, and we computed $u_{j i k}=\cos \theta_{j i k}$ for all the angles $\theta_{j i k}$ formed by these nearest neighbor bonds. The probability distribution $P(u)$ computed at high IL concentration (see Figure 12) provides a baseline to identify characteristic features in systems of lower IL concentration. Moreover, we know that the probability distribution $P(u)$ is independent of $u$ for Gaussian chains, while dipolar chains display a higher proportion of low $u$ angles $(\theta \approx \pi)$, consistently with the long persistence length of dipolar polymers. Comparison of $P(u)$ computed at high IL concentration $(x=0.6)$ and at low IL concentration $(x=0.10)$ displays a clear enhancement of $P(u)$ for negative $u$, that is, for $\theta>\pi / 2$, pointing to a rising proportion of nearly linear structures. The effect is more apparent if we consider only 2-fold coordinated ions, neglecting the ions of higher coordination that are present at all concentration, each contributing a large number of angles. In such a case (see the inset Figure 12), the distribution $P(u)$ has a clear peak at low $u$ values. These observations support the intuitive interpretation of filaments as arising from dipolar interactions.

The analysis of the first coordination shell can be extended to the time domain by monitoring the rate at which ions interchange their closest partners. This is done by identifying all the neighbors within $R_{\mathrm{c}}$ of each ion at time $t_{0}=0$, and computing the average number $\langle n(t)\rangle$ of ions still belonging to the same coordination shell at any later time $t$. The results are plotted on a logarithmic scale in Figure 11a. For $t \geq 200 \mathrm{ps,}$ the simulation data are well approximated by the exponential law $n(t)=n_{0} \exp [-t / \tau(x)]$ that identifies activated processes. The time constants $\tau(x)$ are reported in Figure $11 \mathrm{~b}$ as a function of composition $x$. Starting from a remarkably high value $(\tau=$ $1.8 \mathrm{~ns}$ ) for $x=1, \tau(x)$ decreases monotonically with decreasing $x$ reaching the value $\tau=0.9 \mathrm{~ns}$ at $x=0.2$. Then, it rises again rapidly at lower concentration, providing further evidence of the marked change of coordination and bonding taking place for $0 \leq x \leq 0.2$.

A limited exploration of the effect of temperature on the ionicmolecular equilibrium has been performed by simulating the $x$ 


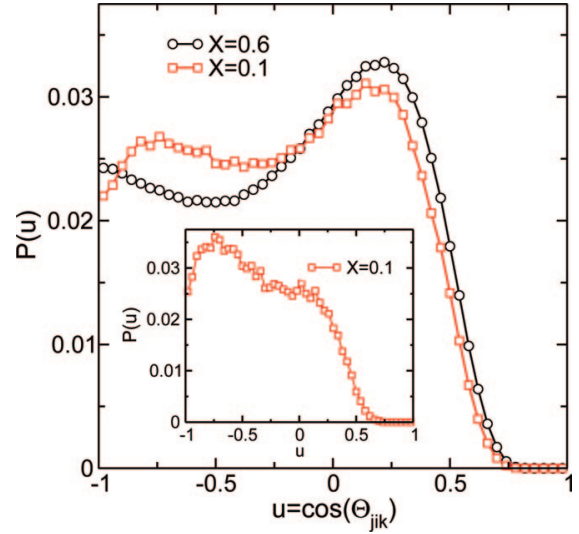

Figure 12. Probability distribution for the variable $u=\cos \theta$, where $\theta$ are the angles between the bonds joining each ion to its nearest neighbours computed at high and low IL concentration. (Inset) Same probability distribution computed by considering only 2 -fold coordinated ions.

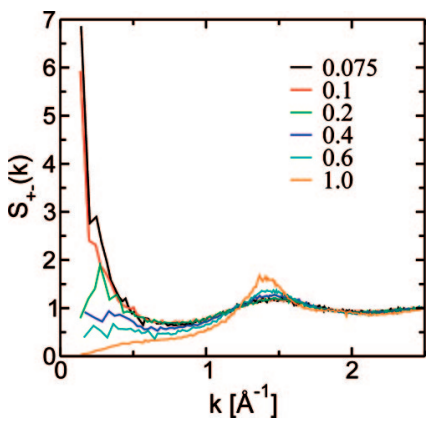

Figure 13. Structure factor $S_{+-}(k)$ for the centres of the $[\mathrm{bmim}]^{+}$and $\left[\mathrm{PF}_{6}\right]^{-}$ion species. Apart from trivial shifts and scale factors, $S_{+-}(k)$ is the Fourier transform of the radial distribution functions in Figure 8.

$=0.2$ sample (which is still apparently ionic) at $T=400$, that is, $50 \mathrm{~K}$ higher than in all other simulations. The effect of this fairly small temperature variation is, as expected, correspondingly small. We find, in particular, that the structure does not change, and transport coefficients display a trivial temperature dependence, and both the diffusion coefficients and the ionic conductivity rise with increasing $T$. We conclude, therefore, that the slight thermal expansion $(100 \Delta V / V=4.2 \%$ in our simulation) caused by the $50 \mathrm{~K}$ rise in temperature does not affect much the system behavior, and only the large variations of the ionic volume obtained by mixing with naphthalene are able to promote the observed sizable change of bonding at the fairly low temperature of our simulations.

It might be useful at this point to discuss the signature of the polar to ionic transition on quantities that can be measured by structure-sensitive experiments such as neutron or X-ray scattering. The Fourier transform of the radial distribution functions of Figure 8 provides a new set of functions $S_{\alpha \beta}(k)(\alpha, \beta=$ $[\mathrm{bmim}]^{+},\left[\mathrm{PF}_{6}\right]^{-}$, or naphthalene), that can be identified with the structure factors of ions and molecules centers (see Figure 13). The onset of the nanometric structures (chains, aggregates and naphthalene clusters) described above are reflected in the growth of a prominent $\operatorname{peak}^{47}$ at $k \leq 0.5 \AA^{-1}$ for IL concentrations $x \leq 0.2$. The height and shape of the peak, and, even more, its dependence on the system size ${ }^{49}$ provide the most

(47) The pre-peak in $S_{+-}(k)$ contrasts with the behaviour of the chargecharge structure factor $S_{Q Q}(k)=S_{++}(k)+S_{-}(k)-2 S_{+-}(k)$, which vanishes $\sim k^{2}$ in the $k \rightarrow 0$ limit. precise information on the transformation taking place in the system. We verified, for instance, that the peak at low $k$ in the $S_{\alpha \beta}(k)$ 's of Sample 1 in Table 2. is nearly indistinguishable from the one computed for Sample II, whose size is twice as large. This provides a quantitative confirmation of the structural similarity of the two samples, and supports the conclusion that the transformation of the bonding character in $\left[b_{m i m}\right]\left[\mathrm{PF}_{6}\right]$ is accompanied by the formation of nanometric patterns in the liquid structures (mesophase), without a macroscopic phase separation.

Unfortunately, the $S_{\alpha \beta}(k)$ functions defined in terms of the ion and molecular centers are only a computational tool introduced to highlight the structure of the liquids, and are not directly accessible to experiments. For instance, the intensity $I(k)$ scattered at angle $\theta$ (with $k=4 \pi \sin (\theta / 2) / \lambda$, where $\lambda$ is the neutron wavelength) during a neutron scattering experiment can be predicted easily from our simulations by computing first the Fourier transform of the atom-atom pair distribution functions, and then combining them with the scattering lengths for the different nuclei reported by standard tables. ${ }^{36}$ The $I(k)$ computed in this way, however, does not show any anomaly at low $k$ when approaching the ionic to dipolar transition, despite the major structural change taking place in the simulated samples. This observation can be explained by the low contrast between [bmim $]\left[\mathrm{PF}_{6}\right]$ and naphthalene when probed by neutron irradiation. Moreover, it is consistent with the results of neutron scattering experiments on a closely related system, that is, [b$\mathrm{mim}]\left[\mathrm{PF}_{6}\right] /$ benzene mixtures, whose scattering intensity is smooth, or shows only small anomalies, in the vicinity of a miscibility gap whose location and size was revealed by thermodynamics studies. ${ }^{48}$

The transformation in the bonding character, and the related change in the structure might be seen more easily by X-ray scattering, exploiting the $k$ dependence of the atomic form factors, or the large cross section of phosphorus atoms. In all cases, however, the determination of the scattering intensity, defined by the weighted average over all the scattering centers in the sample, might not be the easiest route to unravel the complex structure of $[\mathrm{bmim}]\left[\mathrm{PF}_{6}\right] /$ naphthalene mixtures. Other experimental approaches, including NMR, vibrational, and a variety of electron spectroscopies might provide a better way to probe and characterize the geometry and bonding of these mixtures across the ionic to dipolar transition.

\section{Summary and Conclusions}

Structural and dynamical properties of $[\mathrm{bmim}]\left[\mathrm{PF}_{6}\right] /$ naphthalene mixtures have been computed by MD simulations based on an empirical force field. The composition, density, and temperature $(T=350 \mathrm{~K})$ of the simulations closely match those of experimental measurements performed to provide a comprehensive and consistent set of data for the viscosity and ionic conductivity of these mixtures.

The conductivity per ion computed and measured in our study both exhibit a maximum at composition $x \approx 0.15$. The peak in $\sigma(x) / x$ contrasts markedly with the near monotonic increase of the viscosity with increasing IL concentration over the full composition range from pure naphthalene to pure $[\mathrm{bmim}]\left[\mathrm{PF}_{6}\right]$. The composition dependence of conductivity and viscosity highlighted in our study closely correspond to those found in

(48) For example: Deetlefs, M.; Hardacre, C.; Nieuwenhuyzen, M.; Sheppard, O.; Soper, A. K. J. Phys. Chem. B 2005, 109, 1593-1598. (49) Camp, P. J.; Patey, G. N. Phys. Rev. E 2000, 62, 5403-5408. 
previous studies ${ }^{16,18}$ and might represent a fairly general feature of IL mixtures.

The analysis of the configurations generated in our simulations shows that the conductivity maximum is due to a qualitative change in the bonding character of $[\mathrm{bmim}]\left[\mathrm{PF}_{6}\right]$ in solution, which transforms from ionic to molecular with decreasing IL concentration. As expected, the transformation in the bonding character is fairly sharp but continuous; it arises from a gradual shift of the chemical equilibrium between ionic and molecular species and is driven mainly by potential energy considerations.

In a fairly broad composition range around the conductivity maximum, the ionic and molecular phases appear to coexist, giving rise to a complex structure. We find, in particular, that the residual ionic fraction forms nanometric droplets, whereas the neutral ion pairs condense into molecular-thin chains that stretch between ionic droplets and sometimes fold into blobs also of nanometric dimension. All of the IL domains found by simulation in samples of low IL concentration are connected and percolate throughout the simulated samples. This observation is consistent with the fact that $[\mathrm{bmim}]\left[\mathrm{PF}_{6}\right] /$ naphthalene mixtures have nonvanishing ionic conductivity down to the lowest concentration considered in our measurements and computations. No significant variation in the naphthalene structure is observed in the $0 \leq x \leq 0.5$ composition range, supporting the simplifying assumption that naphthalene provides the nearly inert filling that allows a wide variation of the ion density, without the additional complication of strong interactions with the ions.
More in general, and beyond the (already broad) interest in ILs, the results of our simulations provide a direct view of the transformation from ionic liquid to a molecular fluid that is expected to take place in a wide variety of Coulomb systems. Then, the vast number of ILs and solvent combinations that are available provide an invaluable opportunity to investigate the transition at near ambient conditions and fairly high IL concentrations.

Ion aggregation, discussed here in the case of $\left[\mathrm{bmim}^{-}\left[\mathrm{PF}_{6}\right]\right.$, is expected to play an important role also for applications involving ILs and their mixtures with organic solvents of low dielectric constant. On the one hand, the transition marks the stability boundary of the IL phase and might limit the composition range that can be considered for specific applications. On the other hand, the transition provides a further handle to monitor and to change the properties of IL samples. In this respect, the relative sharpness of the transition represents an additional advantage that might allow a precise control of the system state and behavior.

Acknowledgment. We thank Dr. Carlos Pinilla for significant help in the preliminary stages of the present study and for the computation of the atomic charges for the naphthalene force field. We thank Professor R. M. Lynden-Bell for useful discussions and for a careful reading of the manuscript.

Supporting Information Available: Discussion of the interatomic potentials; details on the computation of diffusion coefficients, conductivity, and viscosity. This material is available free of charge via the Internet at http://pubs.acs.org.

JA710841N 